\title{
Electronic properties of the ternary system $\mathrm{YbPd}_{2} \mathrm{Ge}_{2-x}$
}

\author{
V.N. Nikiforov $^{1}$, V.V. Pryadun ${ }^{1}$, A.V. Gribanov ${ }^{1}$, V.Yu. Irkhin² ${ }^{2}$ \\ ${ }^{1}$ Moscow State University, 119899 Moscow, Russia and \\ ${ }^{2}$ M. N. Mikheev Institute of Metal Physics, 620108 Ekaterinburg, Russia
}

\begin{abstract}
The data on the electronic and magnetic properties of the ternary ytterbium-based alloy $\mathrm{YbPd}_{2} \mathrm{Ge}_{1.7}$ are presented and compared with those for $\mathrm{YbPd}_{2} \mathrm{Ge}_{2}$ and $\mathrm{YbPd}_{2} \mathrm{Ge}$ compounds. $\mathrm{YbPd}_{2} \mathrm{Ge}_{1.7}$ demonstrates a moderate heavy-fermion behavior with linear specific heat coefficient $\gamma \simeq 100 \mathrm{~mJ} / \mathrm{mol} \mathrm{K}{ }^{2}$. Magnetic susceptibility is large and demonstrates nearly Curie behavior. Resistivity shows a power-law $\mathrm{T}^{1.2}$-dependence at not too low temperatures $T$. Thermoelectric power has non-monotonous behavior and changes its sign at low $T$.

PACS numbers: $75.30 . \mathrm{Mb}, 71.28 .+\mathrm{d}$
\end{abstract}

\section{INTRODUCTION}

After investigation of unconventional electronic properties of classical heavy-fermion and Kondo-lattice systems during 1980-s, ternary compounds are extensively studied with especial attention to magnetism [1]. Of particular interest are intermetallic ytterbium-based systems which demonstrate a number of peculiarities. In some ytterbium compounds, manifestations of the Kondo effect coexist with ferro- and antiferromagnetic ordering. In a number of ytterbium systems nearly temperature-linear resistivity is observed, indicating non-Fermi-liquid (NFL) behavior. It is interesting that this feature of resistivity is observed in the system $\mathrm{YbMn}_{2} \mathrm{Sb}_{2}$ with nonmagnetic ytterbium, where normal Kondo effect should be absent. These anomalies may be associated with scattering on pseudospin degrees of freedom [2].

Nikiforov et al [3, 4] investigated the ytterbium-based ternary compounds $\mathrm{YbPd}_{2} \mathrm{Ge}, \mathrm{YbPd}_{2} \mathrm{Si}$, YbPdGe, YbPdSi, YbPtGe, synthesized for the first time, and also previously known compounds $\mathrm{YbPd}_{2} \mathrm{Si}_{2}$ and $\mathrm{YbPd}_{2} \mathrm{Ge}_{2}$, the latter one demonstrating superconductivity [5]. From galvanomagnetic properties and magnetic susceptibility it was found that germanium compounds $\mathrm{YbPdGe}$, YbPtGe and $\mathrm{YbPd}_{2} \mathrm{Ge}$ exhibit ferromagnetic ordering at low temperatures (near 16, 10 and $12 \mathrm{~K}$, respectively). Later magnetic and thermodynamic properties were studied thoroughly. In Ref. [6] the values of the Curie temperature $T_{C}=5.4 \mathrm{~K}$, and linear specific heat coefficient $\gamma=209 \mathrm{~mJ} / \mathrm{mol} \mathrm{K}^{2}$ below $T_{C}$ were found for YbPtGe. The magnetic entropy at $T_{C}$ is strongly reduced (which is characteristic for Kondo lattices) and makes up $\mathcal{S}=0.52 R \ln 2$ ( $R$ is the universal gas constant). For YbPdGe, the values $T_{C}=11.4 \mathrm{~K}, \mathcal{S}=0.7 R \ln 2$ and $\gamma=150 \mathrm{~mJ} / \mathrm{mol} \mathrm{K}{ }^{2}$ were obtained [7]. The situation in these systems resembles $\mathrm{CeRuSi}_{2}$ where ferromagnetic ordering coexists with the Kondo effect too [8].

The paper [9] discussed various Kondo manifestations in YbPdGe and YbPtGe, including logarithmic contribution to the resistivity, and a comparison of the transport properties with the corresponding cerium systems CePdGe and $\mathrm{CePtGe}$ (formally trivalent ions of cerium, and ytterbium are similar because correspond to electrons or holes in 4f-shell). As shown in Refs. [10], CeTX compound ( $\mathrm{T}=\mathrm{Pd}$ and $\mathrm{Pt}, \mathrm{X}=\mathrm{Ga}$, Ge and Sn) are antiferromagnetic Kondo systems at low temperatures, in contrast with YbTGe. In the review paper [1], a difference between these cases is discussed in detail for the example of $\mathrm{YbRh}_{2} \mathrm{Si}_{2}$ which is a typical antiferromagnetic heavy fermion ytterbium compound. Despite similarities with $\mathrm{CeRh}_{2} \mathrm{Si}_{2}$ (similar temperatures Kondo $T_{K}$ and resistivity to high amplitude, $T$-linear resistivity above the Neel temperature $T_{N}$ ), there are notable differences, which can be related to more localized features (smaller hybridization with conduction electrons), as well as to a strong spin-orbit interaction in the case of $4 \mathrm{f}$-orbitals of ytterbium.

YbPdSi is a ferromagnetic heavy-fermion system with $T_{C}=8 \mathrm{~K}$ and $\gamma \simeq 300 \mathrm{~mJ} / \mathrm{mol} \mathrm{K}^{2}$ [12]. Studying YbTGe compounds ( $\mathrm{T}=\mathrm{Rh}, \mathrm{Cu}, \mathrm{Ag})\left[13\right.$ showed that YbRhGe is an antiferromagnet with $T_{N}=7 \mathrm{~K}, \mathrm{YbCuGe}$ is a ferromagnet with the moment $0.7 \mu_{B}$ and $T_{C}=8 \mathrm{~K}$; YbAgGe was found to have a very large $\gamma=570 \mathrm{~mJ} / \mathrm{mol} \mathrm{K}^{2}$. In YbAgGe, canted low-temperature magnetic ordering was found [14]. According to [15], YbAgGe is a frustrated heavy fermion antiferromagnet with a complex magnetic phase diagram and NFL behavior.

In the present paper we discuss the electronic and magnetic properties of the ternary ytterbium-based alloy $\mathrm{YbPd}_{2} \mathrm{Ge}_{1.7}$.

*Electronic address: Valentin.Irkhin@imp.uran.ru 


\section{EXPERIMENTAL}

The sample had the mass $m=16.3 \mathrm{mg}$ and the size of $0.8 \times 0.7 \times 1.0 \mathrm{~mm}^{3}$. The temperature dependence of magnetic moment of the sample was measured by a SQUID magnetometer of Quantum Design MPMS- 5 .

The measurements of transport properties were carried out over temperature range $1.5-390 \mathrm{~K}$ by the Quantum Design Physical Property Measurement System. This approach provided simultaneous measurements of electrical resistivity, thermal conductivity and Seebeck coefficient (thermopower at zero electric current) by monitoring both the temperature and voltage drop across a sample as a heat pulse is applied to one end. Electrical resistance was measured employing standard four-contact geometry at a constant current. Measurements were performed under high vacuum (about $10^{-4}$ torr) using a four-probe lead configuration. The Seebeck coefficient was measured when they were disconnected from the current source. The heat flow in the samples was produced by an electrical heater. The thermally induced voltage was measured through the same potential contacts used in measuring resistance. The temperature drop along the sample was measured using the differential manganin-constantan thermocouple.

The thermal conductivity was measured by the method of the longitudinal stable state. The temperature difference across the samples was produced by the electrical heater. The sample was clamped to the other end of the copper base. Heat losses from the sample were minimized by evacuating the chamber. The temperature of the copper base was obtained using a chromelconstantan thermocouple. The temperature difference across the samples was measured by the differential manganinconstantan thermocouple. Since both manganin and constantan have low values of thermal conductivity, this thermocouple did not disturb significantly the heat flow pattern in the sample.

\section{RESULTS FOR ELECTRONIC AND MAGNETIC PROPERTIES}

Magnetic moment of the sample $M$ at $T=2 \mathrm{~K}$ in the field of $9000 \mathrm{G}$ is practically saturated and makes up about 0.02 emu. Magnetic susceptibility demonstrates nearly Curie behavior (Fig.1). The effective magnetic moment per Yb atom $\mu_{\text {eff }}$ determined from the fit $M=N H \mu_{\text {eff }}^{2} / 3 k_{B} T$ ( $H$ is magnetic field, $N$ is the number of atoms, $k_{B}$ is the Boltzmann constant) makes up about $1 \mu_{B}$.

The low-temperature $T$-linear electronic specific heat was obtained by the standard extrapolation starting from temperatures above $20 \mathrm{~K}$ to low temperatures, as shown in Fig. 2. The coefficient $\gamma \simeq 100 \mathrm{~mJ} / \mathrm{mol} \mathrm{K}^{2}$ is markedly enhanced in comparison with standard metals.

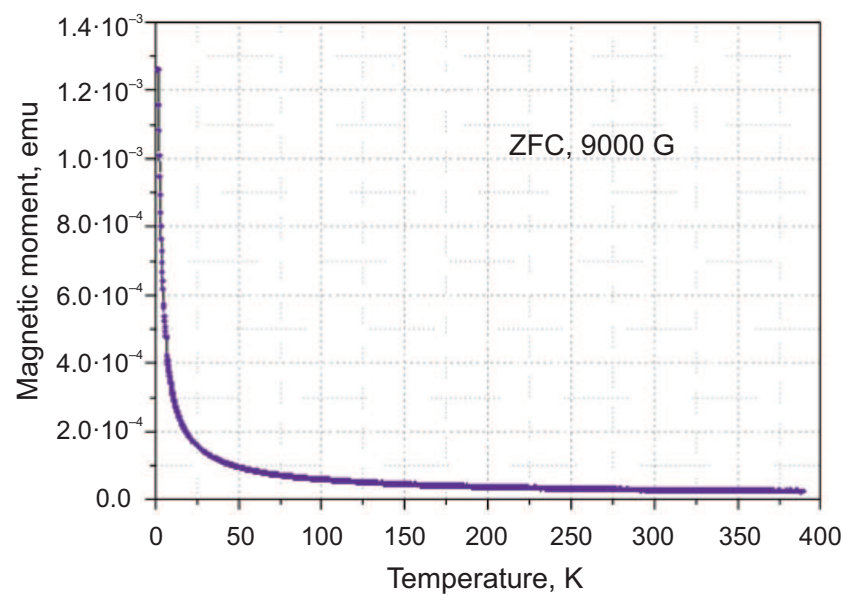

FIG. 1: The temperature dependence of magnetic moment of the sample

At increasing temperature, resistivity $\rho$ shows a power-law increase from the residual value $\rho_{0}=1.3 \mu \Omega \cdot \mathrm{m}$ (see Fig. 3). At not too low temperatures it deviates from the $T^{2}$ behavior, so that we have $\rho-\rho_{0} \sim T^{1.2}$, which indicates nonFermi liquid features. At the same time, we did not found negative magnetoresistance characteristic for incoherent Kondo scattering (see, e.g., the results of Ref. [16] for $\mathrm{YbNi}_{2} \mathrm{~B}_{2} \mathrm{C}$ ) in the field of $9 \mathrm{~T}$ starting from low $T$ up to room temperatures.

Thermal conductivity $\kappa$ (Fig. 4) increases linearly at low temperatures, then grows slowly and sharply increases again. The room temperature values of $\kappa$ of order of $10 \mathrm{~W} / \mathrm{m} \cdot \mathrm{K}$ as well as further increase with temperature are usual for Yb- and Ce-based systems [17, 18]. 


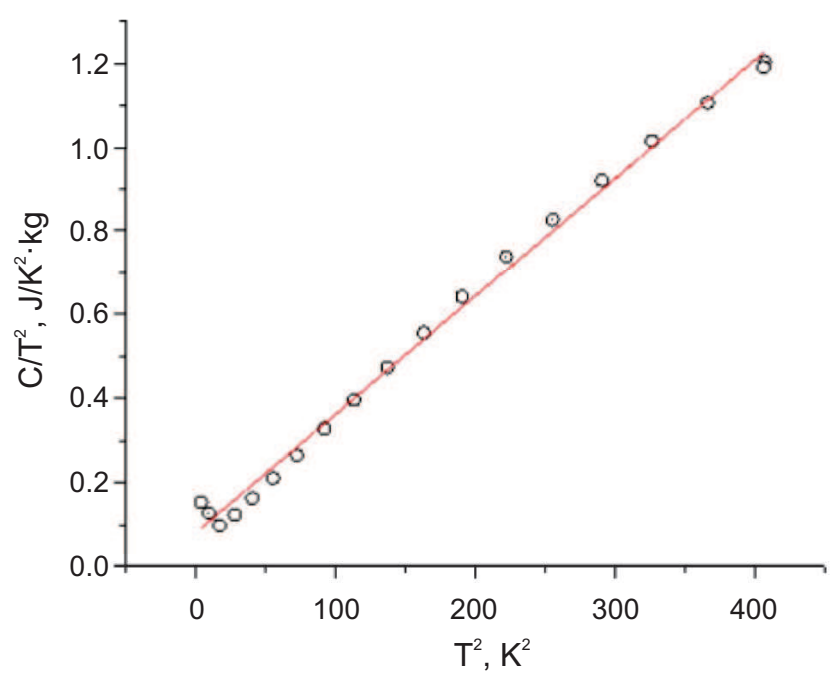

FIG. 2: The determination of $T$-linear electronic specific heat

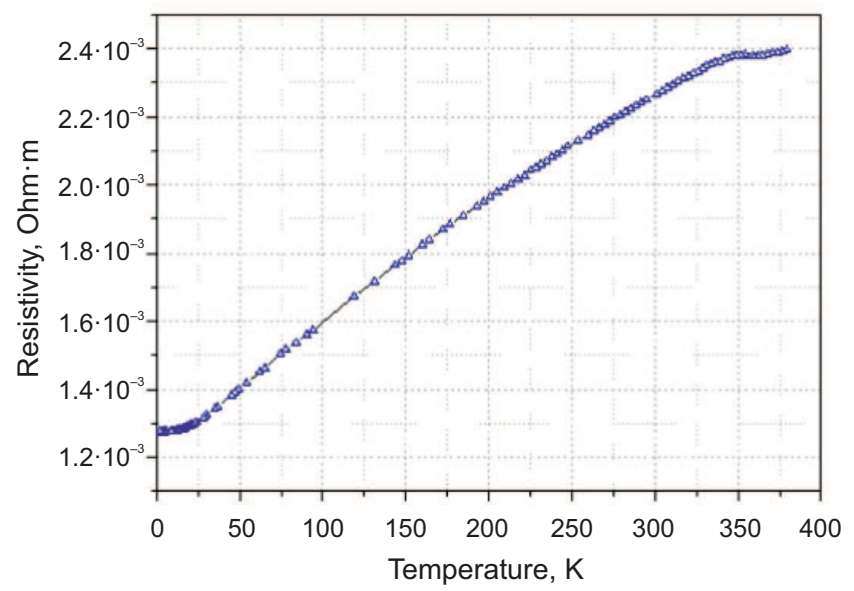

FIG. 3: The temperature dependence of resistivity in a wide temperature region

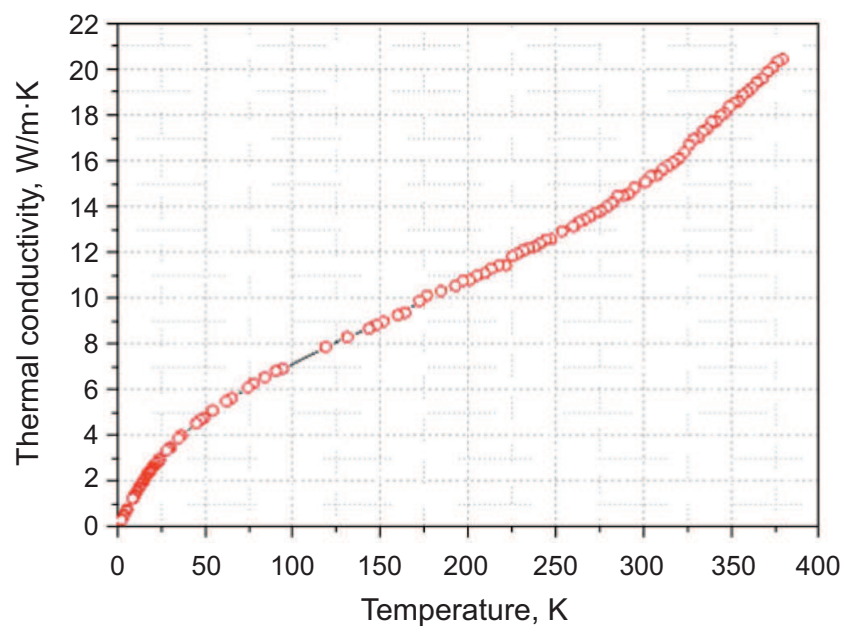

FIG. 4: The temperature dependence of thermal conductivity 


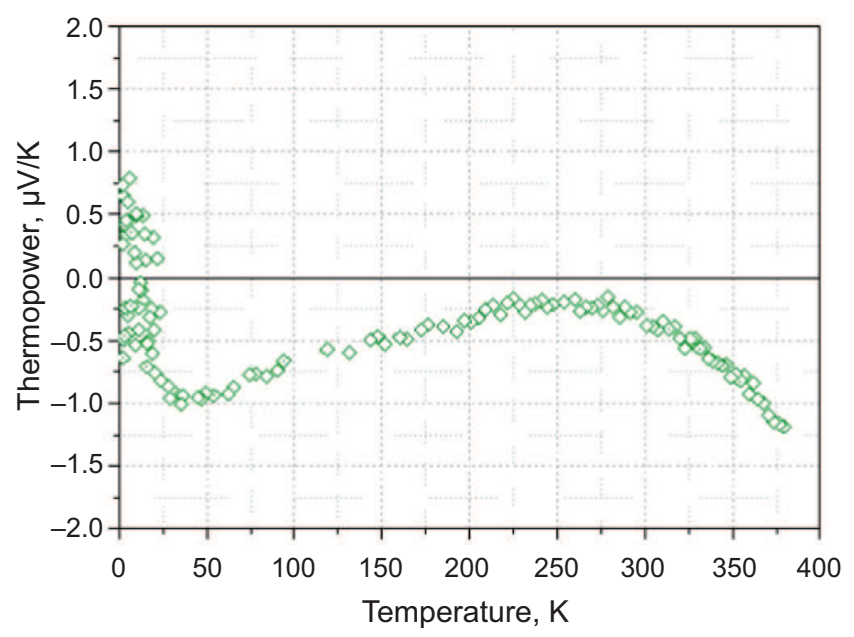

FIG. 5: The temperature dependence of thermoelectric power

Thermoelectric power $S$ has a non-monotonous behavior and changes its sign at low temperatures (Fig. 5). The room temperature values of order of $1 \mu V / K$ are typical for Yb-based systems like $\mathrm{YbCu}_{2} \mathrm{Sb}_{2}$, but are small in comparison with anomalous Ce systems (cf. Ref. [17]). At the same time, much larger absolute values of $S$ are observed in skutterudite systems $\mathrm{Yb}_{3} \mathrm{Co}_{4} \mathrm{Ge}_{13}$ and $\mathrm{Yb}_{3} \mathrm{Co}_{4} \mathrm{Sn}_{13}[18]$.

\section{DISCUSSION}

In intermetallic compounds including $\mathrm{Ce}$ and $\mathrm{Yb}$ elements the hybridization between the $4 \mathrm{f}-$ and itinerant conduction-band electrons induces the instability of magnetic moments and charge configurations. In a number of Yb-based compounds, pressure-induced magnetic-nonmagnetic transitions were observed. The key point here is that $\mathrm{Yb}$ ions fluctuate between the nonmagnetic $\mathrm{Yb}^{2+}(J=0)$ and magnetic $\mathrm{Yb}^{3+}(J=7 / 2)$ states. Since the ionic volume for the $\mathrm{Yb}^{3+}$ state is smaller than for $\mathrm{Yb}^{2+}$, applying pressure or chemical pressure stabilizes the former configuration and induces the appearance of a magnetically ordered state, in contrast to Ce systems.

In this connection, our results on the system $\mathrm{YbPd}_{2} \mathrm{Ge}_{2-x}$ can be treated in terms of the chemical pressure which changes with changing the germanium content. In particular, unlike ferromagnetic compound $\mathrm{YbPd}_{2} \mathrm{Ge}[3]$, the system $\mathrm{YbPd}_{2} \mathrm{Ge}_{1.7}$ shows a nonmagnetic ground state, as well as $\mathrm{YbPd}_{2} \mathrm{Ge}_{2}$. Thus the system passes a magnetic instability (quantum critical point), the ground state being determined by the competition between the magnetic RKKY interaction and the Kondo screening of magnetic moments. The data on specific heat of $\mathrm{YbPd}_{2} \mathrm{Ge}_{1.7}$ demonstrate a moderate heavy-fermion behavior, although the factor $\gamma \simeq 100 \mathrm{~mJ} / \mathrm{mol} \mathrm{K}^{2}$ is somewhat smaller in comparison with other anomalous $\mathrm{Yb}$ systems (see the Introduction).

The deviation from the $T^{2}$ Fermi-liquid behavior of resistivity seems to be more pronounced as compared to $\mathrm{YbPd}_{2} \mathrm{Ge}_{2}$, but weaker than in $\mathrm{YbPd}_{2} \mathrm{Ge}$ [3]. The unusual behavior of resistivity can be ascribed to closeness to the quantum critical point. Sign change in the the thermoelectric power at low temperatures can be related to Kondo features (development of a coherent state) [19].

The authors are grateful to Yu.A. Koksharov for useful discussions and to A.V. Zarubin for the help in preparing the manuscript. The research was carried out within the state assignment of FASO of Russia (theme "Quantum" No. AAAA-A18-118020190095-4) and supported in part by Ural Division of RAS (project no. 18-2-2-11).

[1] V. Yu. Irkhin, Phys. Usp. 60, 747 (2017)

[2] V.N. Nikiforov. V.V. Pryadun, A.V. Morozkin, and V.Yu. Irkhin, Phys. Lett. A 378, 1425 (2014)

[3] V.N. Nikiforov, M.V. Kovachikova, A.A. Velikhovskii, Yu.V. Kochetkov, J. Mirkovic, O.L. Borisenko, and Yu.D. Seropegin, Fiz. Tverd. Tela, 36, 471 (1994)

[4] Yu.D. Seropegin, O.L. Borisenko, O.I. Bodak, V.N. Nikiforov, M.V. Kovachikova, and Yu.V. Kochetkov, Journ. Alloys and Compounds 216, 259 (1994)

[5] G. W. Hull, J. H. Wernick, T. H. Geballe, J. V. Waszczak, and J. E. Bernardini Phys. Rev. B 24, 6715 (1981)

[6] K. Katoh, S. Nakagawa, G. Terui, and A. Ochiai, J. Phys. Soc. Jpn. 78, 104721 (2009) 
[7] K. Enoki, Y. Hirose, S. Yoshiuchi, K. Sugiyama, F. Honda, T. Takeuchi, M. Hagiwara, K. Kindo, E. Yamamoto, Y. Haga, R. Settai, and Y. Onuki: J. Phys. Soc. Jpn 81, SB056 (2012)

[8] V. N. Nikiforov, M. Baran, A. Jedrzejczak, and V. Yu. Irkhin, European Phys. Journ. B86, 238 (2013)

[9] Y. Itoh and H. Kadomatsu, Journ. Alloys and Compounds 280, 39 (1998)

[10] J. Sakurai, Y. Yamaguchi, S. Nishigori, T. Suzuki, and T. Fujita, Journ. Magn. Magn. Mater. 9091, 422 (1990)

[11] J. Flouquet, In: Progress in Low Temperature Physics, Ed. W.P. Halperin, Elsevier, Amsterdam, 2005

[12] N. Tsujii and H. Kitazawa, Solid State Comm. 159, 65 (2013)

[13] K.Katoh, Y.Mano, K.Nakano, G.Terui, Y.Niide and A.Ochiai, Journ. Magn. Magn. Mater. 268, 212 (2004)

[14] P. Bonville, M. Rams, K. Krolas, J.-P. Sanchez, P. C. Canfield, O. Trovarelli, and C. Geibel, Eur. Phys. J. B 55, 77 (2006)

[15] G. M. Schmiedeshoff, E. D. Mun, A. W. Lounsbury, S. J. Tracy, E. C. Palm, S. T. Hannahs, J.-H. Park, T. P. Murphy, S. L. Budko, and P. C. Canfield, Phys. Rev. B 83, 180408(R) (2011)

[16] A. Yatskar, C. H. Mielke, P. C. Canfield, A. H. Lacerda, and W. P. Beyermann Phys. Rev. B 60, 8012 (1999)

[17] V. N. Nikiforov, A. V. Morozkin, and V. Yu. Irkhin, Phys. Metals Metallography 114, 654 (2013); V. N. Nikiforov, V. Yu. Irkhin, and A. V. Morozkin, arXiv:1212.4995

[18] A. V. Morozkin, V. Yu. Irkhin, and V. N. Nikiforov, Journ. Alloys Compounds 549, 121 (2013)

[19] V. Yu. Irkhin and M. I. Katsnelson, Z. Phys. B 75, 67 (1989) 